\title{
The Wolf-Rayet features and mass-metallicity relation of long-duration gamma-ray burst host galaxies
}

\author{
X. H. Han ${ }^{1,2,3,4}$, F. Hammer ${ }^{2}$, Y. C. Liang ${ }^{3}$, H. Flores ${ }^{2}$, M. Rodrigues ${ }^{2}$, J. L. Hou ${ }^{1}$, and J. Y. Wei ${ }^{3}$ \\ 1 Key Laboratory for Research in Galaxies and Cosmology, Shanghai Astronomical Observatory, the Chinese Academy of Sciences, \\ 80 Nandan Road, Shanghai 200030, PR China \\ e-mail: hxh@nao.cas.cn \\ 2 GEPI, Observatoire de Paris-Meudon, Meudon 92195, France \\ e-mail: francois.hammer@obspm.fr \\ 3 National Astronomical Observatories, Chinese Academy of Sciences, Beijing 100012, PR China \\ ${ }^{4}$ Graduate School of the Chinese Academy of Sciences, Beijing 100049, PR China
}

Received 12 May 2009 / Accepted 6 January 2010

\section{ABSTRACT}

\begin{abstract}
Aims. We gather optical spectra of 8 long-duration GRB host galaxies selected from the archival data of VLT/FORS2. We investigate whether or not Wolf-Rayet (WR) stars can be detected in these GRB host galaxies. We also estimate the physical properties of GRB host galaxies, such as metallicity.

Methods. We identify the WR features in these spectra by fitting the WR bumps and WR emission lines in blue and red bumps. We identify the subtypes of the WR stars, estimate the numbers of stars in each subtype, and calculate the WR/O star ratios. The $(\mathrm{O} / \mathrm{H})$ abundances of GRB hosts are inferred from both the electron temperature $\left(T_{\mathrm{e}}\right)$ and the metallicity-sensitive strong-line ratio $\left(R_{23}\right)$, for which we break the $R_{23}$ degeneracy. We compare the environments of long-duration GRB host galaxies with those of other galaxies in terms of their luminosity (stellar mass)-metallicity relations $\left(L-Z, M_{*}-Z\right)$.

Results. We detect WR stars in 5 GRB host galaxies with spectra of relatively high signal-to-noise ratios $(S / N)$. In the comparison of $L-Z, M_{*}-Z$ relations, we show that GRB hosts have lower metallicities than other samples of comparable luminosity and stellar mass. The presence of WR stars and the observed high WR/O star ratio, together with the low metallicity, support the "core-collapsar" model and imply that we are witnessing the first stage of star formation in the host regions of GRBs.
\end{abstract}

Key words. gamma-ray burst: general - stars: Wolf-Rayet - galaxies: abundances - galaxies: fundamental parameters

\section{Introduction}

Gamma-ray bursts (GRBs), the most energetic events in the Universe, were discovered accidentally in the 1960s (Klebesadel et al. 1973). Since then, GRBs have been the targets of intense research. However, the mechanism behind these bursts and the identity of their progenitors remain disputed.

The durations and spectral properties of GRBs suggest a classification of short (of duration $\leqslant 2 \mathrm{~s}$ and hard spectrum) and long bursts (of duration $\geqslant 2 \mathrm{~s}$ and soft spectrum) (Kouveliotou et al. 1993; Hartmann 2005). Short GRBs are believed to originate from the merger of compact binaries such as double neutron star binaries (NS-NS) and black hole-neutron star binaries (BH-NS). For long GRBs, the favored "core-collapsar" model starts from the idea of a rapidly rotating, massive star that has undergone extreme gravitational collapse and formed a central black hole (Woosley et al. 1993; MacFadyen \& Woosley 1999; Klose et al. 2004). According to the collapsar model, the prompt energetic structure of a long GRB is the result of energy dissipation by internal, relativistic shocks, which may last seconds or minutes, at a radius of about $10^{14} \mathrm{~cm}$ from the center of the collapsed star (Hartmann 2005, and references therein). Moreover, the association between GRBs and supernovae $(\mathrm{SNe})$ indicates that in many cases the parent $\mathrm{SN}$ population of GRBs is formed by peculiar type Ibc SNe.

Wolf-Rayet (WR) stars are naturally considered to be the most favored candidates of long duration GRB progenitors. In the stellar evolution model of Hirschi et al. (2005), WR stars, which are massive short-lived stars, satisfy the main criteria for GRB production of black hole formation, loss of hydrogen-rich envelope, and sufficient angular momentum to form an accretion disk around the black hole. According to this model, a lower limit to the metallicity of subsolar value (typically between $Z_{\mathrm{SMC}}$ and $Z_{\mathrm{LMC}}$, i.e., $Z \sim 0.2-0.4 Z_{\odot}$ ) is also a criterion for GRB production. However, magnetic-field breaking poses some difficulties in producing GRBs (Petrovic et al. 2005). To solve this problem, one possible scenario involves assuming that the star of lower metallicity rotates so rapidly that mixing occurs and the star chemically evolves homogeneously without a hydrogen envelope. Moreover, its lower metallicity (typically $Z \lesssim 0.05 Z_{\odot}$ ) causes low mass loss and therefore the retention of a high angular momentum. Those conditions are necessary to produce a GRB (Woosley \& Heger 2006; Yoon \& Langer 2005). Therefore, it is imperative to confirm the presence of WR stars and determine more accurately metallicities in the region of GRBs.

The subtype and number of WR stars, and the relative $\mathrm{WR} / \mathrm{O}$ star number ratio, are related to the star-forming activity and the starburst duration in galaxies. We can achieve a deeper understanding of the evolutionary paths of long-duration GRB progenitors by detecting the WR populations within GRB host galaxies. Moreover, WR evolutionary models suggest that metallicity, one of the important diagnostics of the evolutionary histories of galaxies, affects the properties of WR stars 
(Schaerer \& Vacca 1998). Crowther \& Hadfield (2006) investigated the effect of metallicity on the WR/O star ratio. They found that the WR/O star ratio decreases with metallicity. However, high WR/O star ratios are found in host galaxies of both GRB 980425 and GRB 020903, which have low metallicities (Hammer et al. 2006). The WR/O star ratio-metallicity relation for other GRB hosts is still unknown.

The first detection of a counterpart to a GRB at optical and X-ray was achieved on 28 February 1997 (van Paradijs et al. 1997). Since the discovery of GRB afterglows, GRB host galaxies and their redshifts have been identifiable. Between 1997 and 2007, 588 GRBs were detected. Among them, 325 GRBs had X-ray afterglows, 220 GRBs had optical afterglows, and 55 GRBs had radio afterglows ${ }^{1}$.

The study of GRB host galaxies is very important to the understanding of the physical properties of GRB regions and the nature of GRB progenitors. Although a large number of host galaxies can be identified, most of them are too faint to be observed even using the largest telescopes in the world. So far, only a few dozens of host galaxies have been spectroscopically observed (e.g., GHostS). Moreover, the number of GRB hosts that have been intensively studied spectroscopically is even less.

Evidence from photometric and spectroscopic observations shows that the host galaxies of long-duration GRBs are mostly faint, blue, low-mass, star-forming galaxies with low metallicities (Sokolov et al. 2001; Le Floc'h et al. 2003; Fynbo et al. 2003; Courty et al. 2004; Prochaska et al. 2004; Christensen et al. 2004; Chary et al. 2002; Gorosabel et al. 2005; Fynbo et al. 2006; Wiersema et al. 2007; Kewley et al. 2007; Levesque et al. 2009; Savaglio et al. 2009, hereafter Savaglio09). In contrast, the host galaxies of short-duration GRBs mostly have higher luminosities and higher metallicities than long-duration GRB hosts (Berger 2008).

We study a sample of 8 long-duration GRB hosts with high quality spectroscopic observations. Firstly, we try to detect the WR features in these GRB hosts then study the physical properties of GRB host galaxies, such as their metallicities and luminosity (stellar mass)-metallicity relations $\left(L-Z, M_{*}-Z\right)$. This paper is organized as follows. The sample selection and flux measurements are performed in Sect. 2. In Sect. 3, we describe the identification of the WR features in the spectra of GRB hosts. In Sect. 4, the physical properties of GRB host galaxies are discussed. The discussion and conclusions are presented in Sect. 5. Throughout the paper, we adopt the $\Lambda \mathrm{CDM}$ cosmological model $\left(H_{0}=70 \mathrm{~km} \mathrm{~s}^{-1} \mathrm{Mpc}^{-1}, \Omega_{M}=0.3\right.$, and $\left.\Omega_{\Lambda}=0.7\right)$, and the initial mass function (IMF) proposed by Salpeter (1955). All comparisons performed in this work include a normalization to the same $\Lambda$ CDM cosmological model and IMF. All magnitudes in this paper are in the Vega system.

\section{Data reduction and measurements}

\subsection{The sample selection and data reduction}

One of the main goals of this work is to find evidence of WR stars in GRB hosts by means of optical spectroscopic analysis. We searched archival data of VLT/FORS2 2 to obtain the spectra of GRB host galaxies at $z<1$, which ensures that a sufficient number of emission lines are included in the spectral coverage. These spectra taken with the 600B, 600RI, 600Z grisms

\footnotetext{
1 For the most complete list of GRBs, see the URL: http://www. mpe.mpg.de/ jcg/grbgen . html, maintained by J. Greiner.

2 http://archive.eso.org/eso/eso-archive-main.html
}

Table 1. Basic information of the GRB host galaxies.

\begin{tabular}{cccccc}
\hline \hline GRB & RA $^{a}$ & Dec $^{a}$ & $z$ & $E_{\mathrm{G}}(B-V)^{b}$ & Type \\
\hline GRB 980703 & $23: 59: 07$ & $08: 33: 36$ & 0.966 & 0.061 & long \\
GRB 990712 & $22: 31: 50$ & $-73: 24: 29$ & 0.433 & 0.033 & long \\
GRB 020405 & $13: 58: 03$ & $-31: 22: 22$ & 0.691 & 0.055 & long \\
GRB 020903 & $22: 49: 25$ & $-20: 53: 59$ & 0.251 & 0.033 & long \\
GRB 030329 & $10: 44: 50$ & $21: 31: 17$ & 0.168 & 0.025 & long \\
GRB 031203 & $08: 02: 28$ & $-39: 51: 04$ & 0.105 & 1.040 & long \\
GRB 060218 & $03: 21: 39$ & $16: 52: 02$ & 0.034 & 0.140 & long \\
GRB 060505 & $22: 07: 01$ & $-27: 48: 56$ & 0.089 & 0.021 & long? \\
\hline
\end{tabular}

Notes. (a) Coordinates at the 2000.0 epoch. ${ }^{(b)}$ Galactic extinction (Schlegel et al. 1998).

$(R \sim 1300)$, and $300 \mathrm{~V}$ grism $(R \sim 400)$ of FORS2 were adopted, because they represent a good compromise between relatively high spectral resolution, which allows to resolve the WR features, and relatively high sensitivity, which provides spectra of high quality.

Data reduction and extraction were performed using a set of $\mathrm{IRAF}^{3}$ procedures developed by our team, which can simultaneously reconstruct the spectra and the sky counts of the objects. For some GRBs associated with supernovae, the early-time spectra were carefully checked to avoid the contamination of supernovae, which increases the difficulty in identifying WR features and the error in the emission line flux. Spectra containing contamination by supernovae were then removed.

Wavelength calibration was performed using the HeNeAr lamp spectra. Flux calibration was achieved by observing spectrophotometric stars with the same grism and the same slit width. Galactic extinction was adopted from $\mathrm{NED}^{4}$ and Schaerer \& Vacca (1998). All spectra were transformed into the rest-frame. The spectra taken with different grism, but the same resolution and the same slit width were merged to widen the wavelength coverages (e.g., GRB 980703; GRB 020903). When merging spectra, the exposure times were applied as weighting. The sample has 8 objects including 7 long-duration GRB hosts and 1 possible long-duration GRB host (GRB 060505). The host of GRB 060505 was spatially resolved by VLT/FORS2 spectroscopic observations, therefore the GRB region and the entire host galaxy were studied, separately (see Thöne et al. 2008, for the imaging). The detailed discussion about GRB 060505 is given in Sect. 5

Table 1 lists those 8 GRB hosts in our sample with the name, coordinate at 2000 epoch, redshift, and burst type. The detailed observational information (date, exposure time, seeing, grism, and program) for the objects is given in Table 2.

\subsection{Flux measurements of emission lines}

To measure accurate emission line fluxes, the continuum and absorption lines should be subtracted from the spectrum carefully. To do this, the STARLIGHT ${ }^{5}$, a spectral synthesis code, developed by Cid Fernandes et al. (2005), is used to fit the observed spectrum. STARLIGHT can model the continuum and stellar absorption lines using a linear combination of $N_{*}$ simple

\footnotetext{
3 IRAF is distributed by the National Optical Astronomical Observatories, and is operated by the Association of Universities for Research in Astronomy, Inc., under cooperative agreement with the National Science Foundation.

4 http://nedwww.ipac. caltech.edu/

5 http://www. starlight, ufsc.br
} 
Table 2. Spectroscopic observations of GRB host galaxies from VLT/FORS2.

\begin{tabular}{|c|c|c|c|c|}
\hline UT Date & Exposure time(second) & Seeing $^{a}\left({ }^{\prime \prime}\right)$ & Grism & Program ID \\
\hline \multicolumn{5}{|l|}{ GRB 980703} \\
\hline 2004.07.15-17 & $8 \times 1200$ & 0.75 & $600 \mathrm{Z}$ & 073.B-0482(A) \\
\hline 2004.07.16-17 & $8 \times 1200$ & 0.75 & 600RI & 073.B-0482(A) \\
\hline \multicolumn{5}{|l|}{ GRB 990712} \\
\hline 2005.07.05-06 & $4 \times 1800$ & 0.59 & $300 \mathrm{~V}$ & 075.D-0771(A) \\
\hline \multicolumn{5}{|l|}{ GRB 020405} \\
\hline 2004.07.15-16 & $9 \times 1200$ & 0.70 & 600RI & 073.B-0482(A) \\
\hline \multicolumn{5}{|l|}{ GRB 020903} \\
\hline 2004.07.15 & $6 \times 1200$ & 0.55 & $600 \mathrm{~B}$ & 073.B-0482(A) \\
\hline 2004.07.16 & $6 \times 1200$ & 0.83 & 600RI & 073.B-0482(A) \\
\hline \multicolumn{5}{|l|}{ GRB 030329} \\
\hline 2003.04 .10 & $6 \times 600$ & 1.18 & $300 \mathrm{~V}$ & 071.D-0355(B) \\
\hline 2003.04 .17 & $2 \times 300$ & 0.71 & $300 \mathrm{~V}$ & 071.D-0355(B) \\
\hline 2003.04 .22 & $1 \times 300$ & 0.71 & $300 \mathrm{~V}$ & 071.D-0355(B) \\
\hline 2003.05 .01 & $2 \times 600$ & 0.51 & $300 \mathrm{~V}$ & 071.D-0355(B) \\
\hline 2003.06.19 & $3 \times 900$ & 0.75 & $300 \mathrm{~V}$ & 271.D-5006(A) \\
\hline \multicolumn{5}{|l|}{ GRB 031203} \\
\hline 2003.12 .20 & $2 \times 2700$ & 0.50 & $300 \mathrm{~V}$ & 072.D-0480(A) \\
\hline 2004.09 .20 & $2 \times 1800$ & 0.60 & $300 \mathrm{~V}$ & 073.D-0255(A) \\
\hline 2005.03 .28 & $4 \times 1200$ & 0.69 & $300 \mathrm{~V}$ & 073.D-0418(A) \\
\hline 2005.04.09 & $3 \times 1200$ & 1.26 & $300 \mathrm{~V}$ & 075.D-0771(A) \\
\hline \multicolumn{5}{|l|}{ GRB 060218} \\
\hline 2006.11.27 & $1 \times 3100$ & 0.97 & $300 \mathrm{~V}$ & 078.D-0246(A) \\
\hline $2006.12 .19-20$ & $3 \times 3150$ & 0.72 & $300 \mathrm{~V}$ & 078.D-0246(A) \\
\hline \multicolumn{5}{|l|}{ GRB 060505} \\
\hline 2006.05 .23 & $2 \times 1800+1327+600$ & 0.81 & $300 \mathrm{~V}$ & 077.D-0661(B) \\
\hline
\end{tabular}

Notes. ${ }^{(a)}$ Seeing is the average value in the observing night.

stellar population (SSP) templates from the evolutionary population synthesis code of Bruzual \& Charlot (2003). In the fitting, we use a base of 45 SSPs, which includes 15 ages $(0.001$, $0.003,0.005,0.01,0.025,0.04,0.1,0.28,0.64,0.9,1.4,2.5,5$, $10,13 \mathrm{Gyr})$ at 3 metallicities $\left(0.2,1,2.5 Z_{\odot}\right)$. The Galactic extinction law of Cardelli et al. (1989) (CCM) was adopted in the STARLIGHT fitting. An example of spectral fitting is shown in Fig. 1.

Emission line fluxes were measured manually using SPLOT task in IRAF. The errors originated mainly from three sources: the first is the uncertainty in fitting both the continuum and the stellar absorption line; the second is the uncertainty in the flux measurement; the third is the Poisson noises from both sky and objects, which dominate the error budgets. Emission line fluxes corrected for Galactic extinction are given in Table 3.

\subsection{Dust extinction}

The dust extinction can be estimated from the Balmer-line ratios $(\mathrm{H} \alpha / \mathrm{H} \beta, \mathrm{H} \gamma / \mathrm{H} \beta$, and $\mathrm{H} \alpha / \mathrm{H} \gamma)$. In our dust extinction calculation, we adopted Case $\mathrm{B}$ recombination with an electron density of $100 \mathrm{~cm}^{-3}$ and a temperature of $10000 \mathrm{~K}$. The predicted intrinsic ratio is 2.86 for $I_{0}(\mathrm{H} \alpha) / I_{0}(\mathrm{H} \beta)$, and 0.466 for $I_{0}(\mathrm{H} \gamma) / I_{0}(\mathrm{H} \beta)$ (Osterbrock 1989). We applied the Galactic extinction law of CCM for $R_{V}=3.1$ where $R_{V}$ is the ratio of $A_{V}$ to $E(B-V)$ (Seaton 1979). The values of dust extinction are listed in Table 4. The 2 extinction values for each object derived from the ratios of $\mathrm{H} \alpha / \mathrm{H} \beta$ and $\mathrm{H} \gamma / \mathrm{H} \beta$ are consistent with each other. The $E(B-V)$ derived from $\mathrm{H} \alpha / \mathrm{H} \beta$ was adopted in this study when available, because the higher $S / N$ ratios of these two lines can ensure smaller uncertainties in the results. The $E(B-V)$ derived from $\mathrm{H} \gamma / \mathrm{H} \beta$ was adopted for GRB 980703 and 020405 , because

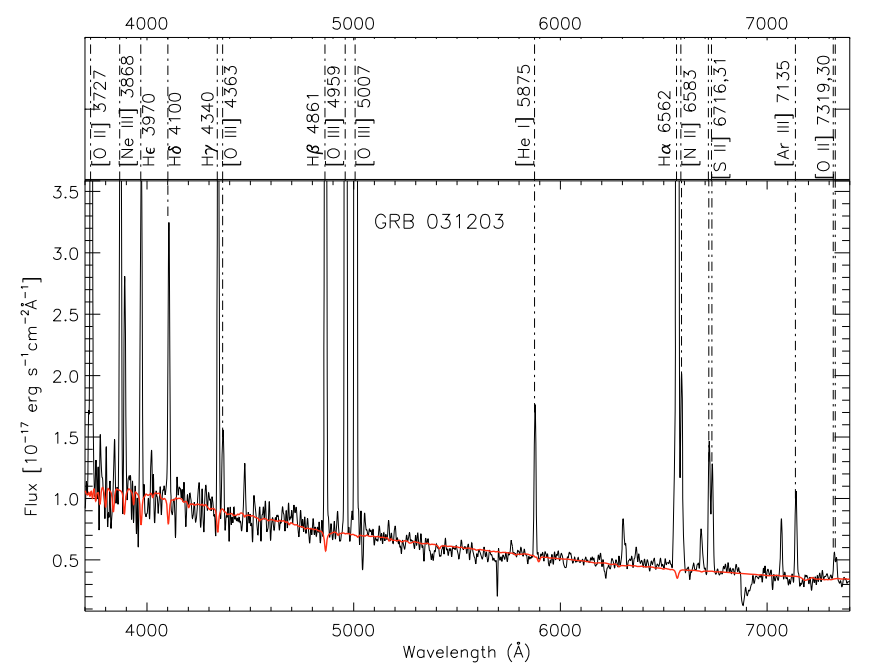

Fig. 1. The spectrum of host galaxy of GRB 031203. The spectral synthesis fitting is plotted in red line. The strong emission lines are marked.

$\mathrm{H} \alpha$ is not available in their spectra. We then corrected emission line fluxes for dust extinction.

\section{Wolf-Rayet bump identification}

The main WR features often seen in optical spectra of galaxies are two characteristic broad emission line clusters. One is in a blue part of the spectrum at around 4600-4680 $\AA$ (hereafter blue bump, Allen et al. 1976; Kunth \& Joubert 1985; Conti 1991; Schaerer \& Vacca 1998). The other is in a red region around 5650-5800 A (hereafter red bump, Kunth \& Schild 1986; Dinerstein \& Shields 1986). The blue bump is actually a 
Table 3. Emission line fluxes of GRB host galaxies (in units of $10^{-17} \mathrm{erg} \mathrm{s}^{-1} \mathrm{~cm}^{-2}$, corrected for Galactic extinction).

\begin{tabular}{|c|c|c|c|c|c|c|c|c|c|}
\hline Ion & 980703 & 990712 & 020405 & 020903 & 030329 & 031203 & 060218 & $060505^{a}$ & $060505^{b}$ \\
\hline$[\mathrm{OII}] \lambda 3727$ & $24.28 \pm 0.11$ & $23.26 \pm 0.15$ & $11.53 \pm 0.16$ & $11.48 \pm 0.36$ & $19.62 \pm 0.32$ & $943.07 \pm 4.80$ & $224.25 \pm 1.01$ & $20.96 \pm 0.82$ & $50.33 \pm 1.88$ \\
\hline $\mathrm{H} \delta$ & $1.66 \pm 0.22$ & $1.95 \pm 0.09$ & - & $1.76 \pm 0.24$ & - & $204.83 \pm 4.41$ & $20.11 \pm 0.57$ & - & - \\
\hline $\mathrm{H} \gamma$ & $3.1 \pm 0.19$ & $4.9 \pm 0.10$ & $1.9 \pm 0.09$ & $3.94 \pm 0.29$ & $4.41 \pm 0.10$ & $499.73 \pm 1.10$ & $42.02 \pm 0.67$ & $3.79 \pm 0.30$ & - \\
\hline [OIII] $\lambda 4363$ & - & $0.92 \pm 0.08$ & - & $0.55 \pm 0.07$ & $0.71 \pm 0.05$ & $79.10 \pm 1.25$ & $4.77 \pm 0.30$ & - & - \\
\hline [FeIII] $] 46658$ & $0.16 \pm 0.09$ & - & - & - & - & - & - & - & - \\
\hline HeII $\lambda 4686$ & - & - & $0.05 \pm 0.03$ & $0.39 \pm 0.06$ & - & $1.20 \pm 0.92$ & $0.81 \pm 0.31$ & - & - \\
\hline [ArIV] $\lambda 4711$ & - & - & $0.07 \pm 0.10$ & $0.80 \pm 0.25$ & - & $4.90 \pm 3.11$ & $2.12 \pm 0.84$ & - & - \\
\hline [ArIV] $\lambda 4740$ & $1.01 \pm 0.05$ & - & - & $0.41 \pm 0.10$ & - & $3.81 \pm 2.91$ & $2.28 \pm 0.91$ & - & - \\
\hline $\mathrm{H} \beta$ & $6.79 \pm 0.30$ & $11.29 \pm 0.07$ & $5.36 \pm 0.10$ & $8.58 \pm 0.29$ & $9.80 \pm 0.16$ & $1135.37 \pm 5.21$ & $91.43 \pm 0.65$ & $8.90 \pm 0.32$ & $21.02 \pm 0.51$ \\
\hline [OIII] $\lambda 4959$ & $4.51 \pm 0.41$ & $17.37 \pm 0.11$ & $5.19 \pm 0.06$ & $15.56 \pm 0.31$ & $11.74 \pm 0.20$ & $2404.78 \pm 5.32$ & $108.4 \pm 0.48$ & $8.47 \pm 0.24$ & $12.47 \pm 0.32$ \\
\hline [OIII] $\lambda 5007$ & $14.39 \pm 0.33$ & $47.28 \pm 0.09$ & $18.33 \pm 0.13$ & $44.11 \pm 0.33$ & $30.53 \pm 0.26$ & $7238.76 \pm 4.68$ & $291.23 \pm 0.66$ & $20.48 \pm 0.48$ & $27.80 \pm 2.30$ \\
\hline $\mathrm{H} \alpha$ & - & $40.34 \pm 0.32$ & - & $26.03 \pm 0.21$ & $30.94 \pm 0.27$ & $3636.63 \pm 4.96$ & $261.51 \pm 0.54$ & $31.49 \pm 0.44$ & $114.8 \pm 3.66$ \\
\hline$[\mathrm{NII}] \lambda 6583$ & - & $<0.41 \pm 0.20$ & - & $0.77 \pm 0.10$ & $0.29 \pm 0.15$ & $109.04 \pm 4.21$ & $10.73 \pm 0.32$ & $1.79 \pm 0.09$ & $29.62 \pm 0.29$ \\
\hline [SII] $\lambda 6716$ & - & - & - & $2.14 \pm 0.17$ & $3.82 \pm 0.09$ & $129.55 \pm 4.33$ & $15.4 \pm 0.36$ & $3.46 \pm 0.13$ & $26.36 \pm 0.51$ \\
\hline [SII] $] 6731$ & - & - & - & $1.28 \pm 0.09$ & $1.89 \pm 0.08$ & $94.63 \pm 2.89$ & $11.56 \pm 0.41$ & $2.80 \pm 0.20$ & $13.98 \pm 0.19$ \\
\hline
\end{tabular}

Notes. ${ }^{(a)}$ GRB site. ${ }^{(b)}$ The entire host galaxy.

Table 4. Dust extinction of GRB host galaxies.

\begin{tabular}{cccccc}
\hline \hline $\mathrm{GRB}$ & $\mathrm{H} \alpha / \mathrm{H} \beta$ & $\mathrm{H} \gamma / \mathrm{H} \beta$ & $A_{V}(\mathrm{H} \alpha / \mathrm{H} \beta)$ & $A_{V}(\mathrm{H} \gamma / \mathrm{H} \beta)$ & $E_{\mathrm{HG}}(B-V)$ \\
\hline 980703 & - & $0.46 \pm 0.23$ & - & $0.14 \pm 0.53$ & $0.05 \pm 0.17$ \\
990712 & $3.57 \pm 0.41$ & $0.43 \pm 0.11$ & $0.55 \pm 0.03$ & $0.50 \pm 0.15$ & $0.18 \pm 0.01$ \\
020405 & - & $0.35 \pm 0.10$ & - & $1.93 \pm 0.36$ & $0.62 \pm 0.12$ \\
020903 & $3.03 \pm 0.91$ & $0.46 \pm 0.32$ & $0.15 \pm 0.09$ & $0.10 \pm 0.57$ & $0.05 \pm 0.03$ \\
030329 & $3.16 \pm 0.57$ & $0.45 \pm 0.12$ & $0.24 \pm 0.05$ & $0.25 \pm 0.20$ & $0.08 \pm 0.02$ \\
031203 & $3.20 \pm 1.79$ & $0.44 \pm 0.25$ & $0.28 \pm 0.04$ & $0.40 \pm 0.12$ & $0.09 \pm 0.01$ \\
060218 & $2.86 \pm 1.64$ & $0.46 \pm 0.34$ & $0.00 \pm 0.02$ & $0.10 \pm 0.12$ & $0.00 \pm 0.01$ \\
$060505^{a}$ & $3.54 \pm 3.59$ & $0.43 \pm 0.72$ & $0.53 \pm 0.08$ & $0.63 \pm 0.35$ & $0.17 \pm 0.03$ \\
$060505^{b}$ & $5.46 \pm 4.51$ & - & $1.60 \pm 0.31$ & - & $0.52 \pm 0.10$ \\
\hline
\end{tabular}

Notes. ${ }^{(a)}$ GRB site. ${ }^{(b)}$ The entire host galaxy.

blend of some broad WR lines, such as N V 4605, $4620 \AA$, N III 4634, $4640 \AA$, C III/C IV 4650, $4658 \AA$, and He II $4686 \AA$, and some nebular emission lines superimposed on the bump, such as [Fe III] $4658 \AA$, He II $4686 \AA$, He I + [Ar IV] $4711 \AA$, and [Ar IV] 4740 (Guseva et al. 2000; Izotov et al. 1998). The red bump is usually much weaker than the blue bump. C IV $5808 \AA$ is commonly seen in the red bump (Kunth \& Schild 1986; Dinerstein \& Shields 1986).

The number of WR stars can be estimated from the luminosity of the blue and red bumps divided by the luminosity of a single WR star in a certain subtype (see, e.g., Guseva et al. 2000). In the following three subsections, we firstly attempt to identify the WR features in our GRB hosts, and then to estimate the subtypes and numbers of WR stars, and finally summarize the results of these GRB host galaxies.

\subsection{Identifying the WR features}

To be able to identify the WR features reliably, we select the high quality spectra of GRB hosts. The criterion for this selection is assumed to be $S / N>10$ in the continuum on both sides of the blue bump to ensure that the WR features detected are real and that a final selected sample is as large as possible. For other cases, there are very few chances to identify the WR features, even if they do exist in the spectra. This final selected sample contains 5 objects: GRB 980703, GRB 020405, GRB 020903, GRB 031203, and GRB 060218.

We identify the WR features of these 5 GRB hosts based on the appearance of the blue bump and WR emission lines by fitting them. This fitting method is also used by Brinchmann et al. (2008). The fitting is performed using the SPECFIT routine in IRAF. We fit the whole blue bump with a single broad Gaussian profile. To fit the WR lines N V 4605, $4620 \AA$, N III $4640 \AA$, C III/C IV 4650, $4658 \AA$, and He II $4686 \AA$, when they exist, we adopt multiple Gaussian profiles to distinguish the broad component, which is mainly from WR stars, from the narrow component, which is mainly from nebulae. Nebular lines, blended within the bump, are also fitted. The FWHMs of narrow components of WR lines are set to be consistent with those of nebular lines. When carrying out the fit, the best-fit continuum is chosen very carefully, because the WR features depend sensitively on the continuum estimates. We limit the overall wavelength shift of the blue and red features to be $|\Delta \lambda| \lesssim 3 \AA$ and the width of the broad component of WR lines to be $\sim 3000 \mathrm{~km} \mathrm{~s}^{-1}$ $F W H M$, which are reasonable values found by (Brinchmann et al. 2008). The fluxes of WR bumps and WR lines are measured from the fitted spectra. The fits are shown in Fig. 2. A significant He I $5875 \AA$ emission line, evidence of young stars such as WR stars, is detected in most of objects.

\subsection{Identifying the subtypes of WR stars and estimating their numbers}

The WR features in galaxies originate mainly in two types of WR stars (WN and WC). In the WN types, the emission lines from helium and nitrogen ions are often seen in the spectra. The emission lines from helium, carbon, and oxygen can be considered as characteristic features of WC types. The relative strengths of these emission lines determine the early (E) and late (L) subtypes of WN stars (WNE, WNL) (e.g., van der Hucht et al. 1981; Conti et al. 1983; Vacca \& Conti 1992), and WC stars (WCE, WCL) (e.g., Torres et al. 1986; Vacca \& Conti 1992). In the blue bump, the broad WR emission lines, such as N V 4605 , $4620 \AA$, N III 4634, $4640 \AA$, C III/C IV 4650, $4658 \AA$, and He II $4686 \AA$ are mainly produced by WNL and WCE stars. In the red bump, C IV $5808 \AA$ is emitted by WCE. Normally, WNE stars cannot be distinguished from other WR stars, since they can emit all these lines. However, their contribution to the bump can be ignored because of their lower luminosity and shorter lifetime than WNL stars. WCL stars can be identified on the basis of their C III 4650, $5696 \AA$ emission lines. Another type of WR star, WO, can be identified using oxygen lines. Subtypes of WO stars can be classified according to the relative strengths of their 
X. H. Han et al.: WR features and $M_{*}-Z$ relation of GRB host galaxies
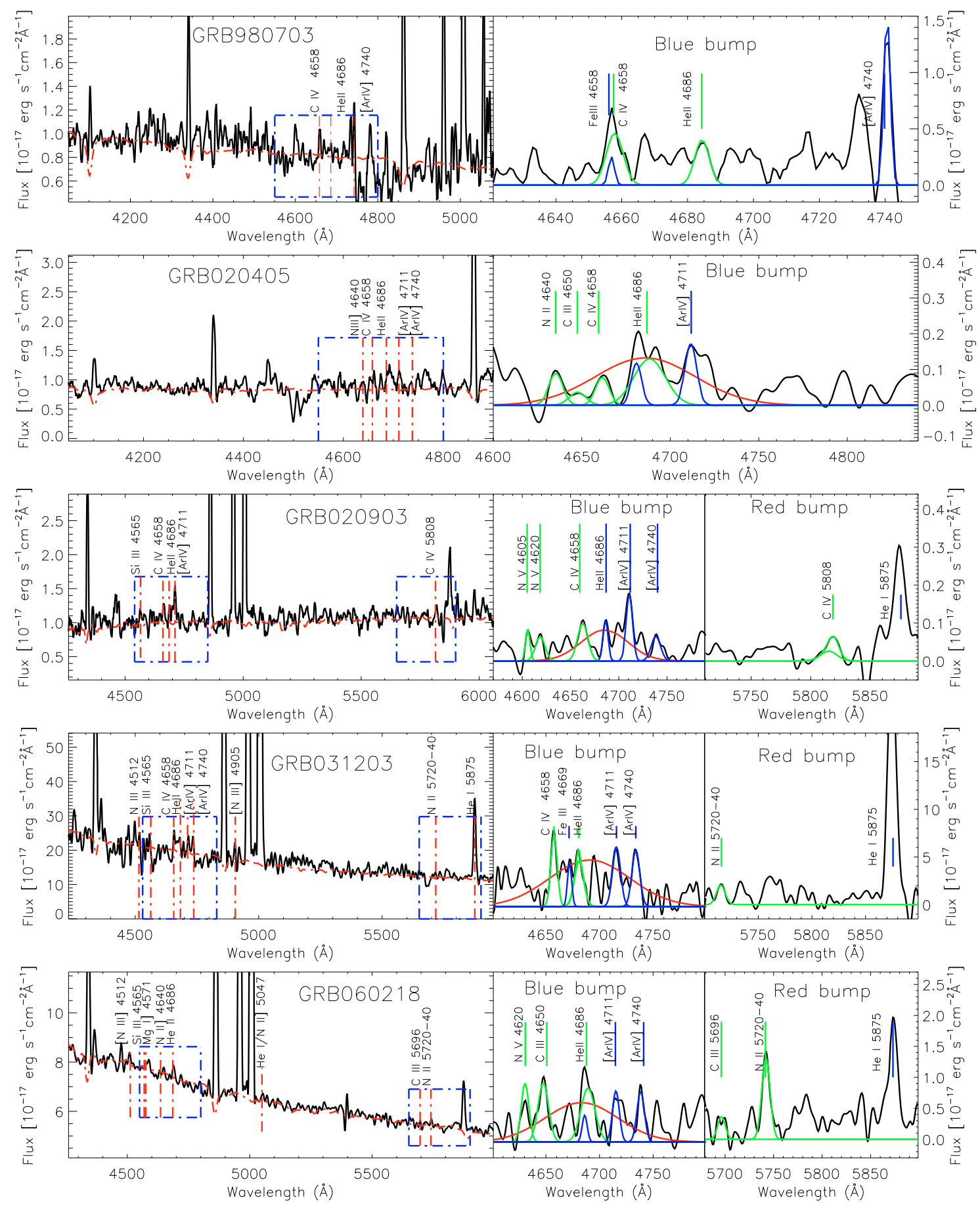

Fig. 2. The spectra of GRB hosts are plotted in the left panels. The continuums are marked using red lines, which are the results of the Starlight fit using BC03 templates. The blue boxes indicate the WR blue and red bumps. The WR emission lines are marked in red dot-dashed lines. In the right panels, the fits of WR bumps are plotted in red profiles. The fits of individual WR line and nebular line are plotted in green and blue profiles respectively (for more details, please see the online color version).

oxygen lines (Barlow \& Hummer 1982; Vacca \& Conti 1992). In our sample, no object exhibits the features of WO stars.

The number of WR stars in a certain subtype can be estimated from the total luminosity of the blue and/or red bumps divided by the luminosity of a single WR star (Guseva et al. 2000). To determine accurately the luminosities of the bumps, the contributions from nebular lines should be subtracted from the bumps.
The number of WCE stars can be derived directly from the luminosity of the red bump, since that luminosity is only produced by WCE stars. WCE stars are usually represented by WC4 stars. The average C IV $5808 \AA$ line luminosity of LMC WC stars is $3.2 \times 10^{36} \mathrm{erg} \mathrm{s}^{-1}$ (Smith et al. 1990; Crowther \& Hadfield 2006).

To determine the number of WNL stars, we consider the luminosity of the blue bump from which we subtract the contribution of WCE stars. The contribution of WCE in the blue bump 
Table 5. Properties of WR stars in GRB hosts.

\begin{tabular}{|c|c|c|c|c|c|c|c|c|c|c|}
\hline \multirow[t]{2}{*}{ Ion } & \multicolumn{2}{|c|}{980703} & \multicolumn{3}{|c|}{020405} & \multicolumn{2}{|c|}{020903} & \multicolumn{2}{|c|}{031203} & 060218 \\
\hline & $F$ & $L$ & $F$ & & $\bar{L}$ & $F$ & $L$ & $F$ & $L$ & $\bar{L}$ \\
\hline blue bump $(\lambda 4650)^{a}$ & - & - & $0.18 \pm 0.09$ & 654.39 & \pm 326.21 & $1.52 \pm 0.31$ & $97.22 \pm 42.90$ & $10.65 \pm 6.06$ & $114.87 \pm 65.37$ & $8.08 \pm 3.25 \quad 6.49 \pm 2.41$ \\
\hline red bump $(\lambda 5008)$ & - & - & - & & - & $0.50 \pm 0.10$ & $32.10 \pm 7.30$ & - & - & - \\
\hline CIII 25696 & - & - & - & & - & - & - & - & - & $3.76 \pm 1.102 .48 \pm 0.76$ \\
\hline$\overline{\mathrm{WNL}}$ & & - & & - & & 2117 & \pm 721 & 5743 & \pm 3268 & $324 \pm 120$ \\
\hline WCE & & - & & - & & 1070 & \pm 210 & & - & - \\
\hline WCL & & - & & - & & & - & & - & $31 \pm 10$ \\
\hline $\mathrm{WR} / \mathrm{O}$ & & - & 0.0 & $4 \pm 0.03$ & & 0.21 & \pm 0.06 & 0.01 & \pm 0.01 & $0.11 \pm 0.04$ \\
\hline
\end{tabular}

Notes. WR emission line fluxes ( $F$, corrected for Galactic extinction) are in units of $10^{-17} \mathrm{erg} \mathrm{s}^{-1} \mathrm{~cm}^{-2}$, Luminosities $(L)$ are in units of $10^{38} \mathrm{erg} \mathrm{s} \mathrm{s}^{-1}$. (a) Fluxes and luminosities of nebular lines were subtracted.

can be estimated from the ratio of the luminosity of WC4 stars in the red bump to that in the blue bump,

$k=\frac{L_{\mathrm{WC} 4}(\lambda 4658)}{L_{\mathrm{WC} 4}(\lambda 5808)}$.

Several values of coefficient $k$ were given by previous works. We adopted the value of $k=1.71 \pm 0.53$ given by Schaerer \& Vacca (1998). WNL stars are usually represented by WN7 stars. The luminosity of a single WN7 in the blue bump, which depends on metallicity, is $2.0 \times 10^{36} \mathrm{erg} \mathrm{s}^{-1}$ for $Z<Z_{\odot}$, or $2.6 \times 10^{36} \mathrm{erg} \mathrm{s}^{-1}$ for $Z>Z_{\odot}$ (Schaerer \& Vacca 1998). The first is adopted in this work, since our samples have lower metallicity than solar (see Sect. 4).

The number of WCL stars can be estimated from the luminosity of C III $5696 \AA$. For a WCL single star, the value of luminosity of C III $5696 \AA$ adopted in this work is $8.1 \times 10^{36} \mathrm{erg} \mathrm{s}^{-1}$ (Schaerer \& Vacca 1998; Guseva et al. 2000). However, the luminosity of this weak line in a single star is still not well known.

The ratio of relative WR/O star number can be estimated by applying two methods to optical spectra. The method developed by Arnault et al. (1989) uses the flux in the entire WR blue bump, while another (Vacca \& Conti 1992) uses only the flux of the broad He II $4686 \AA$ line. We use the first method in this work because it is more suitable for low resolution spectra, for which the broad He II $4686 \AA$ A line cannot be separated clearly from the bump. The formula is

$$
\log \left[\frac{\mathrm{WR}}{(\mathrm{WR}+\mathrm{O})}\right]=(-0.11 \pm 0.02)+(0.85 \pm 0.02) \log \left(\frac{L_{\text {blue bump }}}{L_{\mathrm{H} \beta}}\right) \text {. }
$$

For each GRB host, both the fluxes and luminosities of the WR bumps and other weak WR features are given in Table 5. Fluxes given here are not corrected for interstellar extinction, while luminosities are absolute ones, which are transformed from these fluxes after being corrected for extinction.

Several sources contribute to the uncertainties in estimating the numbers of WR stars. The luminosity of a single WR star adopted is based on the average of LMC and Milky Way WR stars, which could be higher than our host galaxy sample, because of the difference in metallicity. The difference in metallicity could also affect the value of $k$. Besides these two major effects, there are three other sources: the observational uncertainty, the errors in the multi-component profile fit, and the contamination by nebular lines to the blue bump. Together, they produce the measured an uncertainty, assumed to be $30-60 \%$, in the number of WR stars and the WR/O star number ratio.

\subsection{The results}

We present the WR features and the number of WR stars in each of our GRB hosts in detail as follows, and as also given in Table 5.

GRB 980703 - The $S / N$ in continuum on both sides of the blue bump (hereafter $S / N^{*}$ ) is about 12 . We do not detect the convincing WR bump in the spectrum. However, two WR lines, C IV $4658 \AA$ and He II $4686 \AA$ are identified, which may be emitted by WNL and WCE stars (Schaerer \& Vacca 1998; Brinchmann et al. 2008). The red bump is not included within the spectral range of the spectrum. Therefore, we cannot verify the WR star subtypes.

GRB $020405-$ The $S / N^{*}$ is 10 . A blue bump can be seen clearly in the spectrum. Some broad WR lines, N II $4640 \AA$, C III $4650 \AA$, C IV $4658 \AA$, and He II $4686 \AA$ are identified in the bump, which implies the presence of WNL and WCE. The weak C III $4650 \AA$ indicates the presence of WCL stars. The WR subtypes and the number of WR stars cannot be verified since there is no red part to the spectrum.

GRB 020903 - The $S / N^{*}$ is about 16. A strong blue bump is detected in the spectrum. Broad lines N V 4605, $4620 \AA$, C IV $4658 \AA$, and C IV $5808 \AA$ indicate the existence of WNL and WCE stars (Brinchmann et al. 2008). The number of WCE stars is estimated by using the luminosity of the red bump, which can be interpreted as being produced by $1070 \pm 210$ WCE stars. Adopting the value of $k=1.71 \pm 0.53$, the contribution of these WCE stars in the blue bump is $5.49 \pm 1.81 \times 10^{39} \mathrm{erg} \mathrm{s}^{-1}$. After subtracting the contribution of WCE stars, the luminosity of WNL stars in the blue bump is $4.23 \pm 1.46 \times 10^{39} \mathrm{erg} \mathrm{s}^{-1}$, which corresponds to $2117 \pm 721 \mathrm{WNL}$ stars.

GRB $031203-$ The $S / N^{*}$ is about 20. The strong signature of the blue bump is shown in the spectrum. The broad WR lines C IV $4658 \AA$, He II $4686 \AA$ are detected in the blue bump. In the red part, we do not detect the characteristic line of WCE, C IV 5808, which implies that the number of WCE in this galaxy can be ignored. Besides that, the other two WR lines, N III $4905 \AA$, N II 5720-40 are also detected. All of these WR features are indicative of WNL stars (Smith et al. 1996; Guseva et al. 2000; Brinchmann et al. 2008). The number of WNL stars is $1585 \pm 540$ as estimated from the luminosity of the blue bump.

GRB 060218 - The $S / N^{*}$ is higher than 40. Many WR lines are identified, including N III $4512 \AA$, N V $4620 \AA$, N II $4640 \AA$, He II $4686 \AA$, He I/N II $5047 \AA$, and N II 5720-40 ̊. All these lines exhibit the characteristics of WNL stars (Massey 2003; Crowther \& Smith 1997; Guseva et al. 2000; Brinchmann et al. 2008). Two WR lines, C III $4650 \AA$ and C II $5696 \AA$, are also 
identified, which are characteristic of WCL stars (Brinchmann et al. 2008). The presence of WCE stars cannot be confirmed, since no C IV 5808 line is detected. Using the luminosity of the blue bump, we estimate the number of WNL stars to be $324 \pm 120$. The luminosity of C III $5696 \AA$ corresponds to $31 \pm 10$ WCL stars.

The relative WR/O star number ratios are estimated using the luminosities of blue bumps and $\mathrm{H} \beta$. For the hosts of GRB 020405, 020903, 031203, and 060218, the ratios are $0.26 \pm$ $0.08,0.21 \pm 0.06,0.09 \pm 0.02$, and $0.11 \pm 0.04$, respectively.

In summary, obvious WR features are detected in 4 out of 5 spectra of long-duration GRB hosts, except for GRB 980703, which shows no convincing WR bump but two WR lines. Moreover, the numbers of WR stars for 3 GRB hosts are in the range from 300 to 6000 , which is consistent with the typical number of WR stars $\left(\sim 100-10^{5}\right.$, Vacca \& Conti 1992; Guseva et al. 2000) in WR galaxies. High WR/O star number ratios are also estimated in these 4 GRB hosts Therefore, the presence of WR stars in these GRB hosts is verified. However, because of the small sample studied here, the conclusion that WR stars exist in all the long-duration GRB hosts still cannot be confidently made. The results of this work in all cases support the scenario that WR stars are the progenitors of long GRBs, and are consistent with the previous studies described below.

Hammer et al. (2006) first discovered WR stars in three nearby long-duration GRB host galaxies (GRB 980425, GRB 020903, GRB 031203) using deep spectroscopic observations. Since then, little has been done to detect WR features in long-duration GRB hosts. Margutti et al. (2007) claimed that the identification of WR emission lines in GRB 031203 is uncertain. However, their Fig. 3 does show the strong WR blue bump. The N III $4640 \AA$ and C IV $4658 \AA$ blended broad lines can also be identified in the bump, which is not attempted in their work. Wiersema et al. (2007) did not detect the obvious He II $4686 \AA$ and WR bump in GRB 060218. However, we note that the UVES spectra that they used were taken at a time close to the burst, which means that the significant contamination by the associated SN 2006aj cannot be ignored in the WR feature identification. In their work, an upper limit to the ratio $\mathrm{WR} /(\mathrm{WR}+$ O) for the galaxy is estimated to be $<0.4$, which is consistent with the result $(\mathrm{WR} / \mathrm{O}=0.11 \pm 0.04)$ estimated in this work.

\section{The physical properties of GRB host galaxies}

Metallicity is a fundamental parameter for probing the properties of GRB progenitors and the environment of GRB regions. Strong emission lines in spectra of GRB host galaxies allow us to estimate metallicities. The luminosity (stellar mass)-metallicity $\left(L-Z, M_{*}-Z\right)$ relation of galaxies is a fundamental relation for indicating their evolutionary status and star-formation histories. We estimate the metallicities of these GRB hosts, and then study them in terms of the $L-Z$ and $M_{*}-Z$ relations.

\subsection{AGN contamination}

The traditional Baldwin-Phillips-Terlevich (BPT) diagram (Baldwin et al. 1981) can diagnose the origin of the narrow emission lines of emission-line galaxies. Figure 3 shows the locations of GRB host galaxies in the BPT diagram. The [O III]/H $\beta$ vs. $[\mathrm{N} \mathrm{II}] / \mathrm{H} \alpha$ diagnostic lines are taken from Kewley et al. (2001) and Kauffmann et al. (2003). The [O III]/H $\beta$ vs. [S II] $/ \mathrm{H} \alpha$ relation from Kewley et al. (2001) is shown. This illustrates that the

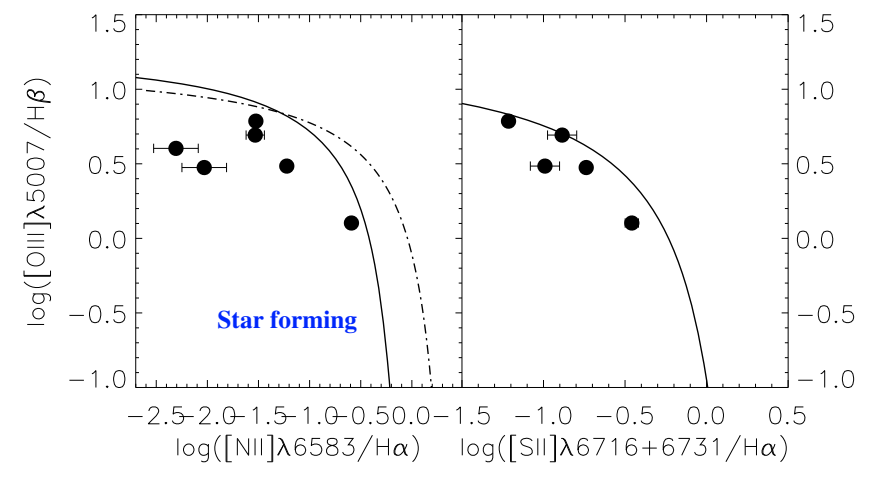

Fig. 3. The diagnostic diagrams of GRB host galaxies. In the left panel, $[\mathrm{O} \mathrm{III}] / \mathrm{H} \beta$ vs. [N II] $/ \mathrm{H} \alpha$ relations is shown. The relation from Kewley et al. (2001) is drawn in the dot-dashed curve. The solid curve is from Kauffmann et al. (2003). [O III]/H $\beta$ vs. [S II] $/ \mathrm{H} \alpha$ relation from Kauffmann et al. (2003) is shown in the right panel.

GRB host galaxies are star-forming galaxies and AGN contamination in GRB host galaxies can be ignored.

\subsection{Metallicity}

In terms of oxygen abundance, the metallicities of GRB hosts can be estimated using the $T_{\mathrm{e}}$ method and $R_{23}$ methods. The $T_{\mathrm{e}}$ method based on electron temperature is a "direct" way. The "indirect" method is the so-called "strong-line" method, such as the $R_{23}$ method based on the empirical relationship between $\mathrm{O} / \mathrm{H}$ and $R_{23}$.

It is well known that the $T_{\mathrm{e}}$ method is effective for metalpoor galaxies because the characteristic line [O III]4363 is only observable in this case. Out of 8 galaxies, 5 GRB hosts (GRB 990712, 020903, 030329, 031203, and 060218) clearly exhibit an [O III]4363 line, which implies that they have low metallicities and lie on the lower-metallicity branch of the $R_{23}$ diagnostic diagram. [N II $] /[\mathrm{O}$ II $]$ also provides a reliable means of locating a galaxy on the metallicity branch. As Kewley et al. (2008) suggested, the galaxies with $\log ([\mathrm{N}$ II]6583/[O II]3727) $\leqslant-1.2$ lie on the lower metallicity branch. All of these 5 objects and the GRB region of GRB 060505 have values of $\log ([\mathrm{N} \mathrm{II}] 6583 /[\mathrm{O}$ II $] 3727) \leqslant-1.2$, while the entire GRB 060505 host galaxy lies on the upper branch, because of the value of its $\log ([\mathrm{N} \mathrm{II}] 6583 /[\mathrm{O}$ II $] 3727) \geqslant-1.2$. The values of $\log ([\mathrm{N}$ II $] 6583 /[\mathrm{O}$ II $] 3727)$ are given in Table 6. For another 2 GRB hosts, 980703, 020405, we cannot verify the metallicity branch which on they lie because their [NII]6583 lines are not detected. Moreover, we use $\log ([\mathrm{N} \mathrm{II}] 6583 / \mathrm{H} \alpha)$ to verify the metallicity branches $(\leqslant-1.3$ for lower branch; $\geqslant-1.1$ for upper branch, Kewley et al. 2008) for the sample. These three indicators provide the consistent results for these galaxies.

For the 5 host galaxies that exhibit the [O III]4363 emission line, we use the $T_{\mathrm{e}}$ method to calculate their oxygen abundances. Electron temperature and electron density are estimated using the TEMDEN task of IRAF, which is based on a 5-level atom program and was described by Shaw \& Dufour (1994). The flux ratios of $[\mathrm{O}$ III $] I(4959+5007) / I(4363)$ are adopted to calculate electron temperature when available. Using the flux ratio of [S II] $I(6716) / I(6731)$, the electron density can be derived, which is needed in the electron temperature and oxygen abundance calculation.

When carrying out the electron density calculation, the [S II] $I(6716) / I(6731)$ doublet is not available or too noisy for some objects in our sample. Therefore, we tested the sensitivities of 
Table 6. Metallicities of GRB host galaxies.

\begin{tabular}{|c|c|c|c|c|c|c|c|c|c|}
\hline & 980703 & 990712 & 020405 & 020903 & 030329 & 031203 & 060218 & $060505^{g}$ & $060505^{h}$ \\
\hline$N_{\mathrm{e}}[\mathrm{SII}]^{a}$ & - & $20 \sim 200$ & - & $20 \sim 200$ & $20 \sim 200$ & $52.63 \pm 43.20$ & $120.68 \pm 32.22$ & - & - \\
\hline$T_{\mathrm{e}}[\mathrm{OIIII}]^{b}$ & - & $16434.9 \pm 425.8$ & - & $13517.1 \pm 335$ & $17911.7 \pm 325.0$ & $12902.2 \pm 305.2$ & $15113.3 \pm 335.5$ & - & - \\
\hline $12+\log (\mathrm{O} / \mathrm{H})(\mathrm{Te})^{\mathrm{c}}$ & - & $7.812 \pm 0.15$ & - & $8.014 \pm 0.15$ & $7.653 \pm 0.14$ & $8.142 \pm 0.07$ & $7.883 \pm 0.09$ & - & - \\
\hline $\begin{array}{l}12+\log (\mathrm{O} / \mathrm{H})^{d} \\
\left(K 99 \_l\right)\end{array}$ & $8.28 \pm 0.14$ & $8.18 \pm 0.12^{f}$ & $8.10 \pm 0.12$ & $8.11 \pm 0.12^{f}$ & $8.06 \pm 0.10^{f}$ & $8.11 \pm 0.11^{f}$ & $8.16 \pm 0.14^{f}$ & $8.06 \pm 0.14^{f}$ & $7.99 \pm 0.09$ \\
\hline $\begin{array}{l}12+\log (\mathrm{O} / \mathrm{H})^{d} \\
\left(K 99 \_u\right)\end{array}$ & $8.44 \pm 0.15$ & $8.47 \pm 0.15$ & $8.53 \pm 0.15$ & $8.50 \pm 0.15$ & $8.56 \pm 0.16$ & $8.51 \pm 0.15$ & $8.50 \pm 0.16$ & $8.57 \pm 0.15$ & $8.66 \pm 0.16^{f}$ \\
\hline $\begin{array}{l}\log ([\mathrm{NII}] 6583 \\
/[\mathrm{OII}] 3727)\end{array}$ & - & -2.36 & - & -1.47 & -2.12 & -1.23 & -1.45 & -1.36 & -0.52 \\
\hline$A_{V}^{e}$ & $0.144 \pm 0.532$ & $0.551 \pm 0.025$ & $1.927 \pm 0.359$ & $0.146 \pm 0.086$ & $0.244 \pm 0.046$ & $0.280 \pm 0.040$ & $0.000 \pm 0.018$ & $0.526 \pm 0.081$ & $11.601 \pm 0.310$ \\
\hline
\end{tabular}

Notes. ${ }^{(a)}$ Electronic density estimated from the [S II] flux ratio: $I(6716) / I(6731) .{ }^{(b)}$ Temperature in K estimated from the following flux ratios: [O III] $I(4959+5007) / I(4363) .{ }^{(c)}$ Metallicity, $12+\log (\mathrm{O} / \mathrm{H})$, estimated using the effective temperature method. ${ }^{(d)}$ Metallicity, $12+\log (\mathrm{O} / \mathrm{H})$, estimated following Kobulnicky et al. (1999). K99_l (their Eq. (8)) is valid for the lower branch; K99_ $u$ (their Eq. (9)) is valid for the upper branch. ${ }^{(e)}$ Extinction coefficient, $A_{V}$ (in magnitude) is derived using the standard Balmer ratio of $\mathrm{H} \alpha$ and $\mathrm{H} \beta$. For GRB 980703 and $020405, A_{V}$ from the ratio of $\mathrm{H} \gamma$ and $\mathrm{H} \beta$ is adopted. ${ }^{(f)}$ Adopted metallicity in this work. The $R_{23}$ degeneracy are broken using the [N II]/H $\alpha$ and [N II]/[O II] ratios. ${ }^{(g)}$ The GRB site. ${ }^{(h)}$ The entire host galaxy.

both electron temperature and oxygen abundance to electron density, finding that neither is very sensitive to electron density. This allows us to estimate oxygen abundance for a wide range of electron density $\left(20-200 \mathrm{~cm}^{-3}\right)$ without significant bias (the discrepancy is less than $5 \%$ ). To calculate $T_{\mathrm{e}}$-based oxygen abundances, we use the methods described by Stasińska (2005), Izotov et al. (2006), Yin et al. (2007), Liang et al. (2006).

The $R_{23}$ method has been extensively discussed in the literature (Pagel et al. 1979; McGaugh 1991; Kobulnicky et al. 1999; Tremonti et al. 2004; Yin et al. 2007; Liang et al. 2007). To estimate their $R_{23}$-based metallicities, we adopt the formulae of Kobulnicky et al. (1999) for both the upper and lower branches of $R_{23}-(\mathrm{O} / \mathrm{H})$ solutions. We use indicators of [N II]6583/[O II]3727 and [N II]6583/H $\alpha$ to break the $R_{23}$ degeneracy. The estimated oxygen abundances and errors are listed in Table 6 . The $T_{\mathrm{e}}$-based and $R_{23}$-based metallicity estimates have an acceptable discrepancy of $0.03-0.3$ dex.

We now compare our metallicity estimates with those in the literature. There are four objects for which $T_{\mathrm{e}}$-based oxygen abundances have been estimated in the literature. For the host of GRB 020903, Hammer et al. (2006) measured a $12+\log (\mathrm{O} / \mathrm{H})_{T_{\mathrm{e}}}$ of 7.97, which is very consistent with this work (8.01) within the errorbars; Savaglio09 gave 8.22, which is 0.21 dex higher. For the host of GRB 030329, Levesque et al. (2009) obtained 7.72 from Keck spectra, while we obtain 7.65. These two estimates are extremely consistent within the errorbars. For the host of GRB 031203, Prochaska et al. (2004) obtained 8.10, Margutti et al. (2007) inferred 8.12, Levesque et al. (2009) obtained 7.96, and Savaglio09 measured 8.02, while we obtain 8.14. All of these estimates are consistent within the errorbars.

For the host of GRB 060218, Wiersema et al. (2007) measured a $12+\log (\mathrm{O} / \mathrm{H})$ value of 7.54, Levesque et al. (2009) obtained 7.62, Savaglio09 measured 7.29, and we inferred 7.88, which is closest to the second value. The discrepancy between these works could be caused by contamination by the associated supernova. Savaglio09 gathered the very early-time spectra observed by Pian et al. (2006) and Sollerman et al. (2006). Wiersema et al. (2007) acquired their spectrum within 1 month of the burst, while our spectrum of the host was taken in Dec. 2006, 10 months after the burst, and Levesque et al. (2009) took the spectrum even later (19 months after the burst). In this case, the contamination of the GRB 060218 host galaxy by the light of SN2006aj was significant and lasted for several months. This contribution strongly affects measurements of emission lines; hence, the errors in the metallicity estimates for the early spectra might be large. Our result is close to that of Levesque et al. (2009), which is also derived from the latetime observation. Anyway, all of them show this host has low metallicity.

We do not compare our $R_{23}$-based estimates with any other work, because different calibrations provide very different values, and we discussed the discrepancy between our own $T_{\mathrm{e}}$ and $R_{23}$-based abundances above.

\subsection{Luminosity vs. metallicity}

We obtain multi-band photometry from literature, which is shown in Table 7. All apparent magnitudes are corrected for Galactic foreground extinction (Schlegel et al. 1998) and internal extinction. The $k$-correction is performed for the magnitudes to $z=0$ by using the $k$ correct v4-1-4 program ${ }^{6}$ (Oke \& Sandage 1968; Hogg et al. 2002; Blanto \& Roweis 2007). The absolute $B$ and $K$-band magnitudes corrected for Galactic extinction are given in Table 8. We plot our long-duration GRB hosts on the $L-Z$ diagram (Fig. 4), which is presented in terms of absolute $B$ magnitude and the $R_{23}$-based metallicities. The magnitude and metallicity of the entire GRB 060505 host galaxy are presented in the $L-Z$ diagram. For GRB 980703, the mean value between the lower and upper branch is adopted, when the $R_{23}$ degeneracy cannot be broken. The $L-Z$ relations of various low and high redshift samples from the literature (SDSS star-forming galaxies from Tremonti et al. (2004), UV-selected galaxies from Contini et al. (2002), large magnitude-limited sample from Lamareille et al. (2004), emission-line-selected galaxies from Melbourne \& Salzer (2002), irregular and spiral galaxies from Kobulnicky \& Zaritsky (1999), and irregular galaxies from Skillman et al. (1989) and Richer \& McCall (1995)) are also shown in Fig. 4. In comparison with the $L-Z$ relation, the GRB hosts show an obvious discrepancy from other samples. GRB hosts have lower metallicity values than other galaxies at given luminosities. For luminous GRB hosts, this discrepancy is even larger. This trend is also shown in Levesque et al. (2009).

\footnotetext{
${ }^{6}$ http://cosmo.nyu.edu/ mb144/kcorrect/
} 
Table 7. Photometry of GRB host galaxies.

\begin{tabular}{|c|c|c|c|c|c|c|c|c|c|}
\hline GRB & $U$ & $B$ & $V$ & $R$ & $I$ & $J$ & $H$ & $K$ & Refs. \\
\hline 980703 & - & $23.40 \pm 0.12$ & $23.04 \pm 0.08$ & $22.58 \pm 0.06$ & $21.95 \pm 0.25$ & $20.87 \pm 0.11$ & $20.27 \pm 0.19$ & $19.62 \pm 0.12$ & $1,2,3$ \\
\hline 990712 & $23.12 \pm 0.05$ & $23.36 \pm 0.09$ & $22.39 \pm 0.03$ & $21.84 \pm 0.02$ & $21.41 \pm 0.03$ & $20.81 \pm 0.17$ & $20.25 \pm 0.19$ & $20.05 \pm 0.10$ & 3 \\
\hline 020903 & - & $21.70 \pm 0.10$ & $20.80 \pm 0.10$ & $20.80 \pm 0.10$ & $20.50 \pm 0.10$ & - & - & - & 4,5 \\
\hline 030329 & $22.68 \pm 0.10$ & $23.42 \pm 0.07$ & $22.88 \pm 0.05$ & $22.80 \pm 0.04$ & - & $21.52 \pm 0.04$ & $21.17 \pm 0.24$ & - & 6 \\
\hline 031203 & $22.41 \pm 0.18$ & $22.32 \pm 0.05$ & $20.53 \pm 0.05$ & $20.44 \pm 0.02$ & $19.40 \pm 0.04$ & $18.28 \pm 0.02$ & $17.78 \pm 0.02$ & $16.54 \pm 0.02$ & 7 \\
\hline 060218 & $20.45 \pm 0.15$ & $20.46 \pm 0.07$ & $20.19 \pm 0.04$ & $19.86 \pm 0.03$ & $19.47 \pm 0.06$ & $18.99 \pm 0.16$ & $18.52 \pm 0.22$ & $18.69 \pm 0.34$ & $8,9,10$ \\
\hline 060505 & $18.43 \pm 0.05$ & $18.89 \pm 0.02$ & $18.27 \pm 0.02$ & $17.90 \pm 0.02$ & $17.51 \pm 0.02$ & $17.29 \pm 0.08^{a}$ & - & $15.85 \pm 0.04$ & 11 \\
\hline & - & - & $\mathrm{F}^{255 \mathrm{~W}^{b}}$ & $\mathrm{~F} 702 \mathrm{~W}^{b}$ & $\mathrm{~F} 14 \mathrm{~W}^{b}$ & - & - & - & \\
\hline 020405 & - & - & $22.63 \pm 0.05$ & $21.84 \pm 0.05$ & $21.29 \pm 0.05$ & - & - & - & 12 \\
\hline
\end{tabular}

References. (1) Vreeswijk et al. (1999); (2) Sokolov et al. (2001); (3) Christensen et al. (2004); (4) Soderberg et al. (2004); (5) Bersier et al. (2006); (6) Gorosabel et al. (2005); (7) Margutti et al. (2007); (8) Sollerman et al. (2006); (9) Castro Cerón et al. (2008); (10) Kocevski et al. (2007); (11) Thöne et al. (2008); (12) Wainwright et al. (2007).

Notes. The magnitudes are not corrected for Galactic extinction. ${ }^{(a)}$ FORS1 $z$ filter (Central wavelength: $9100 \AA$; band width: $1305 \AA$ ). ${ }^{(b)}$ F555W (Central wavelength: $5407 \AA$; band width: $1236 \AA$ ) ; F702W (Central wavelength: $6895 \AA$ A; band width: $1389 \AA$ ); F814W (Central wavelength: $7940 \AA$ A; band width: $1531 \AA$ ).

Table 8. Magnitudes and stellar masses of GRB host galaxies.

\begin{tabular}{lccc}
\hline \hline GRB & $M_{B}$ & $M_{K}$ & $\log M_{*}\left[M_{\odot}\right]$ \\
\hline 980703 & $-20.85 \pm 0.12$ & $-24.41 \pm 0.12$ & $11.14 \pm 0.39$ \\
990712 & $-18.67 \pm 0.09$ & $-21.85 \pm 0.10$ & $10.14 \pm 0.34$ \\
020405 & - & - & - \\
020903 & $-18.96 \pm 0.10$ & - & - \\
030329 & $-16.22 \pm 0.07$ & - & - \\
031203 & $-20.58 \pm 0.05$ & $-22.27 \pm 0.02$ & $10.13 \pm 0.26$ \\
060218 & $-16.02 \pm 0.07$ & $-17.23 \pm 0.34$ & $8.28 \pm 0.55$ \\
$060505^{a}$ & $-19.24 \pm 0.02$ & $-22.20 \pm 0.04$ & $10.18 \pm 0.24$ \\
\hline
\end{tabular}

Notes. Absolute magnitudes $M_{B}$ and $M_{K}$ are corrected for galactic foreground extinction (Schlegel et al. 1998). ${ }^{(a)}$ The magnitudes and stellar mass of the entire galaxy of GRB 060505 host.

\subsection{Stellar mass vs. gas phase metallicity}

The stellar mass of galaxy can be estimated from the stellar mass-to-light ratio and color following Bell et al. (2003). We use the $(B-V)$ color and $M_{K}$ to estimate the stellar masses of GRB host galaxies. The formula is

$\log \left(\frac{M_{*}}{M_{\odot}}\right)=-0.4\left(M_{K}-3.28\right)+\left[a_{K}+b_{K}(B-V)+0.15\right]$,

where $M_{K}$ is the $K$-band absolute magnitude and $(B-V)$ is the rest-frame color. The coefficients $a_{K}$ and $b_{K}$ come from Table 7 of Bell et al. (2003). The stellar masses are listed in the last column of Table 8. We compare our results with previous work on GRB hosts (Chary et al. 2002; Castro Cerón et al. 2006, 2008; Savaglio et al. 2009). Our results are more consistent with the stellar masses derived by Castro Cerón et al. (2008) within the errorbars. However, our results are systematically higher than theirs by $\sim 0.3$ dex. This discrepancy between the two datasets could be caused by the use of different $M_{*} / L_{K}$ ratios. The stellar masses given by Savaglio09 are the lowest of all previous work. Castro Cerón et al. (2008) explain that the reasons for this discrepancy could be the different $M_{*} / L_{K}$ ratios applied and the underestimated dust extinction in Savaglio09, which is also confirmed by our dust extinction (see Table 4).

We plot our long-duration GRB hosts on the stellar massmetallicity $\left(M_{*}-Z\right)$ diagram (Fig. 5$)$. The $T_{\mathrm{e}^{-}}$and $R_{23}$-based metallicities are adopted and plotted in the left and right panels,

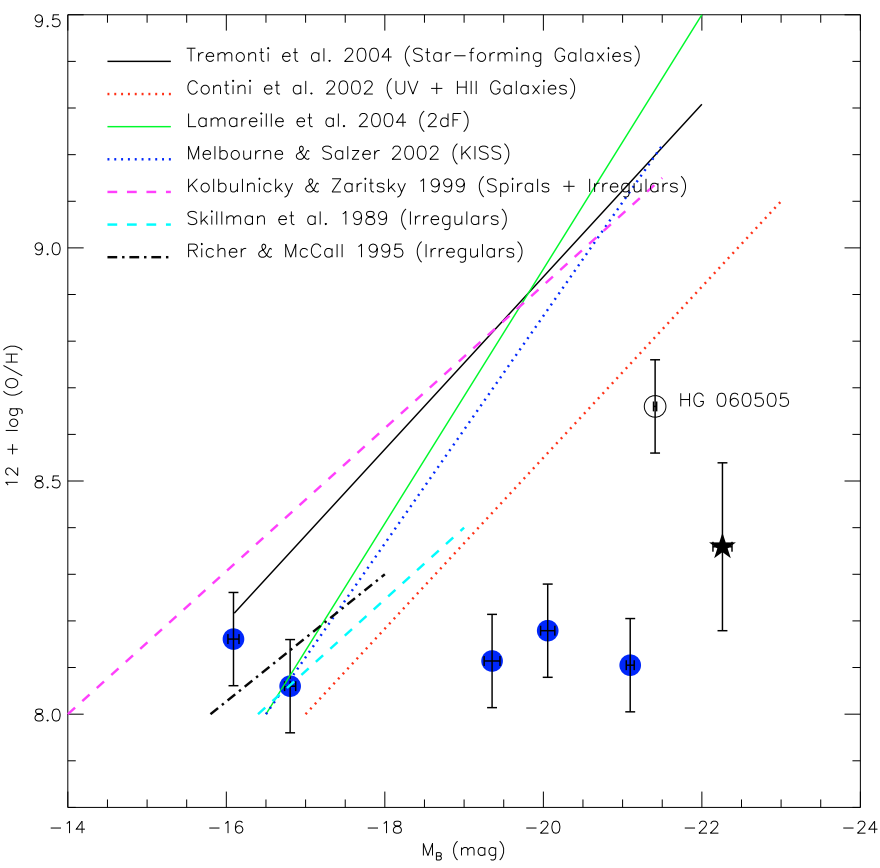

Fig. 4. The $L-Z$ relation of long-duration GRB hosts. The $R_{23}$-based metallicities are adopted. The blue filled circles represent oxygen abundances which break the degeneracy of $R_{23}$. The black star represents the mean value between the lower and upper branch of GRB 980703. The open circle represents the entire host of GRB 060505. Luminositymetallicity relation for SDSS galaxies and various galaxy samples are drawn from the literature (see legend). See the online color version for more details.

respectively. In the right panel, the open circle and black filled star represent the hosts of GRB 060505 (not the GRB region) and GRB 980703, respectively. The $M_{*}-Z$ relation of local normal star-forming galaxies selected from SDSS derived by Liang et al. (2007) is given in Fig. 5 (the red dashed line derived from the $T_{\mathrm{e}}$ method in the left panel; the red dotted line derived from the $R_{23}$ method in the right panel). The $T_{\mathrm{e}^{-}}$and $R_{23}$-based metallicities of long-duration GRB host galaxies are all obviously lower than those of local star-forming galaxies with comparable stellar masses. 


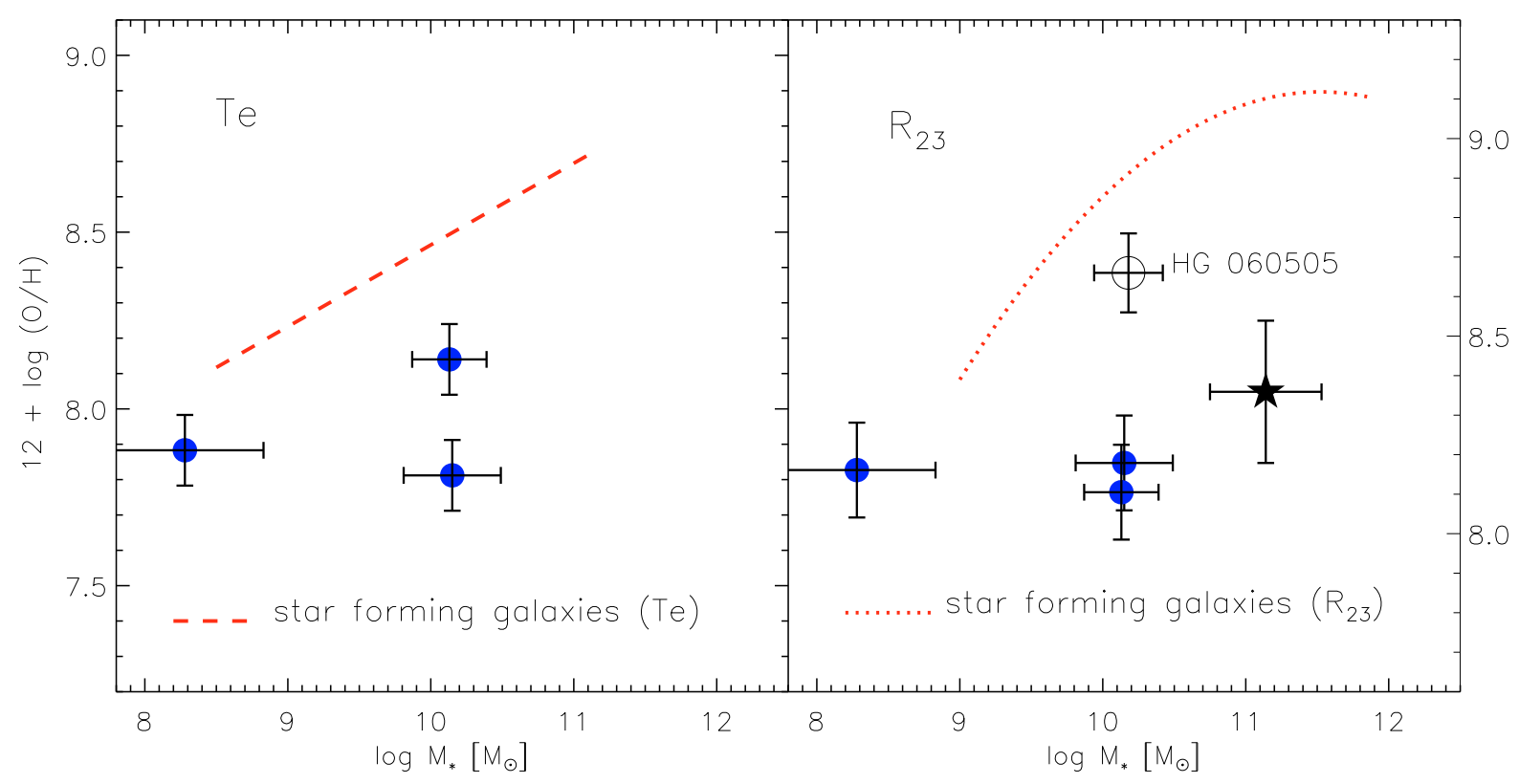

Fig. 5. In the left panel, the $T_{\mathrm{e}}$-based (the filled circles) oxygen abundances of long-duration GRB hosts are given. The red dashed line refers to the $M_{*}-Z$ relation of local star-forming galaxies from SDSS derived from $T_{\mathrm{e}}$ method (Liang et al. 2007). In the right panel, we plot the $R_{23}$-based oxygen abundances of long-duration GRB hosts derived using formulae from Kobulnicky et al. (1999) (the blue filled circles - oxygen abundances that break the degeneracy; the black star - oxygen abundances from mean value between the lower and upper branch). The open circle represents the entire host of GRB 060505. The red dotted line refers to the $M_{*}-Z$ relation of local star-forming galaxies from SDSS derived from $R_{23}$ method (Liang et al. 2007). See the online color version for more details.

\section{Discussion and conclusions}

We have attempted to identify the WR stars in GRB host galaxies. According to the collapsar model, WR stars are considered as the most favored candidates of the progenitor of longduration GRBs, therefore the presence of WR stars in longduration GRB hosts can directly validate this model. We have investigated a sample of $8 \mathrm{GRB}$ host galaxies from archival data from VLT/FORS2. Out of those 8 GRB hosts, 5 galaxies having spectra with $S / N>10$ were for our sample to detect the WR features. The presence of WR features was verified in this entire sample. Out of those 5 GRB hosts, 4 certainly have WR features detected, which are GRB 020903, GRB 031203, GRB 020405, and GRB 060218. For the remaining one, GRB 980703, the detection is marginal. The subtypes and numbers of WR stars in those 5 GRB hosts were also derived. Those results strongly support the collapsar model by illustrating the link between WR stars and GRBs.

Additional aim was to investigate the physical properties of long-duration GRB host galaxies, such as metallicity, luminosity, and stellar mass. Comparing with literature, we used the most consistent measurements and calculation methods for all 8 objects to ensure systematic coherence and comparability. We found that the long-duration GRB hosts show obvious disagreement with the $L-Z$ and $M_{*}-Z$ relations derived for low redshift galaxies in several samples. The luminous and massive longduration GRB hosts have lower metallicities than other galaxies in these samples.

The WR/O star ratio in WR galaxies is found to decrease as a function of decreasing metallicity by Crowther \& Hadfield (2006). The WR/O star ratio and metallicity in GRB host galaxies do not show a clear trend. However, compared to WR galaxies (Guseva et al. 2000), the observed WR/O ratios are higher in the GRB hosts studied here, while metallicities in GRB hosts are obviously lower than in the numerous population of low redshift galaxies. This suggests that the hosted regions of GRBs are consistent with the first stage of star formation in a relatively pristine medium. Furthermore, the ratio of WC/WN stars is also found to decrease with metallicity. The relation between the ratio of WC/WN stars and metallicity was derived by Massey (2003). The hosts of GRB 020903, 060218 deviate from the relation, in having higher $\mathrm{WC} / \mathrm{WN}$ ratios of $\sim 0.5$ and 0.1 than Local Group galaxies (see their Fig. 11, the upper panel). The collapsar model suggests that the WC stars are more likely to be the progenitors of long-duration GRBs than the WN stars. Therefore, the observed high WC/WN ratio could also be evidence to support this model, although the sample is small.

One of our galaxies, the host of GRB 060505, is well resolved in the ground-based observation. However, the type of this burst remains a topic of debate. GRB 060505 has a burst duration of $\sim 4 \mathrm{~s}$, but lacks evidence of an accompanying supernova. It is classified as a long-duration GRBs that have no associated supernovae (e.g., GRB 060614) by Thöne et al. (2008). However, Levesque \& Kewley (2007) suggest that the environment of GRB 060505 is more consistent with the host environments of short-duration GRBs. We have investigated the metallicity of the GRB site and the entire host galaxy separately. We have found a relatively low metallicity in the GRB region and a higher one in the entire host, which are consistent with Thöne et al. (2008). Unfortunately, the $S / N$ of its spectrum is not high enough to detect WR star features. However, a very young ( $\sim 6 \mathrm{Myr}$ ) stellar population in the GRB site is found by Thöne et al. (2008) using stellar population modeling. This low age corresponds to the lifetime of a $32 M_{\odot}$ star. Evidence from the properties of the GRB region suggests that the GRB 060505 originated in a long-duration core-collapse progenitor.

Most of the GRB host galaxies in our sample are too distant to allow spatially resolved analysis with ground-based spectroscopic observations. Therefore, the only information that we can derive concerns about the global properties of galaxies. The properties of the actual explosion sites are still unclear. For 
further study of GRB progenitors and properties of GRB host galaxies, more high-resolution images and deep spectroscopy are needed. X-shooter at the VLT will be the ideal instrument to investigate in greater detail the chemical and stellar population properties of GRBs.

Acknowledgements. We thank the anonymous referee for very helpful comments, which improve well this work. We are very grateful to P. A. Crowther, X. Y. Chen, H. L. Li, J. Wang, L. P. Xin, and W. K. Zheng for very helpful discussions. We acknowledges James Wicker for his careful correcting of English. This work was supported by the Natural Science Foundation of China (NSFC 10933001, 10573028, 10673002, 10803008, 10821061, 10833005); the National Basic Research Program of China (973 Program, 2007CB815402, 2007CB815404, 2007CB815406, 2009CB824800); the Young Researcher Grant of National Astronomical Observatories, Chinese Academy of Sciences; and the Group Innovation Project (10821302). The STARLIGHT project is supported by the Brazilian agencies CNPq, CAPES and FAPESP and by the France-Brizil CAPES/Cofecub program.

\section{References}

Allen, D. A., Wright, A. E., \& Goss, W. M. 1976, MNRAS, 177, 91 Arnault, Ph., Kunth, D., \& Schild, H. 1989, A\&A, 224, 73

Baldwin, A., Phillips, M. M., \& Terlevich, R. 1981, PASP, 93, 817

Barlow, M. J., \& Hummer, D. G. 1982, in Wolf-Rayet Stars: Observations, Physics, and Evolution, ed. C. W. H. de Loore, \& A. J. Willlis (Dordrecht: Reidel), IAU Symp. 99, 387

Bell, E. F., McIntosh, D. H., Katz, N., et al. 2003, ApJS, 149, 289

Berger, E. 2008, ApJ, 690, 231

Bersier, D., Fruchter, A. S., Strolger, L. G., et al. 2006, ApJ, 643, 284

Blanton, M. R., \& Roweis, S. 2007, AJ, 133, 734

Brinchmann, J., Kunth, D., \& Durret, F. 2008, A\&A, 485, 657

Bruzual, G., \& Charlot, S. 2003, MNRAS, 344, 1000

Cardelli, J. A., Clayton, G. C., \& Mathis, J. S. 1989, ApJ, 345, 245

Castro Cerón, J. M., Michalowski, M. J., Hjorth, J., et al. 2006, ApJ, 653, 85

Castro Cerón, J. M., Michalowski, M. J., Hjorth, J., et al. 2008, [arXiv:0803.2235v2]

Chary, R., Becklin, E. E., \& Armus, L. 2002, ApJ, 566, 229

Christensen, L., Hjorth, J., \& Gorosabel, J. 2004, A\&A, 425, 913

Cid Fernandes, R., Mateus, A., Sodré, L., Stasińska, G., \& Gomes, J. M. 2005 MNRAS, 358, 363

Conti, P. S. 1991, ApJ, 377, 115

Conti, P. S., Leep, E. M., \& Perry, D. N. 1983, ApJ, 268, 228

Contini, T., Treyer, M. A., Sullivan, M., et al. 2002, MNRAS, 330, 75

Courty, S., Björnsson, G., \& Gudmundsson, E. H. 2004, MNRAS, 354, 581

Crowther, P. A., \& Hadfield, L. J. 2006, A\&A, 449, 711

Crowther, P. A., \& Smith, L. J. 1997, A\&A, 320500

Dinerstein, H. L., \& Shields, G. A. 1986, ApJ, 311, 45

Fynbo, J. P. U., Jakobsson, P., Möller, P., et al. 2003, A\&A, 406, L63

Fynbo, J. P. U., Watson, D., Thöne, C. C., et al. 2006, Nature, 444, 1047

Gorosabel, J., Jelínek, M., de Ugarte Postigo, A., Guziy, S., \& Castro-Tirado,

A. J. 2005, Nuovo Cimento C, 28, 677

Guseva, N. G., Izotov, Y. I., \& Thuan, T. X. 2000, ApJ, 531, 776

Hammer, F., Flores, H., Schaerer, D., et al. 2006, A\&A, 454, 103

Hartmann, D. H. 2005, Nature, 436, 923

Hirschi, R., Meynet, G., \& Maeder, A. 2005, A\&A, 443, 581

Hjorth, J., Watson, D., Fynbo, J. P. U., et al. 2005, Nature, 437, 859

Hogg, D. W., Blanton, M., Strateva, I., et al. 2002, AJ, 124, 646

Izotov, Y. I., \& Thuan, T. X. 1998, ApJ, 500, 188

Izotov, Y. I., Stasińska, G., Meynet, G., Guseva, N. G., \& Thuan, T. X. 2006, A\&A, 448, 955

Kauffmann, G., Heckman, T. M., Tremonti, C., et al. 2003, MNRAS, 346, 1055
Kewley, L. J., \& Ellison, S. L. 2008, ApJ, 681, 1183

Kewley, L. J., Dopita, M. A., Sutherland, R. S., Heisler, C. A., \& Trevena, J. 2001, ApJ, 556, 121

Kewley, L. J., Brown, W. R., Geller, M. J., Kenyon, S. J., \& Kurtz, M. J. 2007, AJ, 133, 882

Klebesadel, R. W., Strong, I. B., \& Olson, R. A. 1973, ApJ, 182, 85

Klose, S., Greiner, J., Rau, A., et al. 2004, AJ, 128, 1942

Kobulnicky, H. A., \& Zaritsky, D. 1999, ApJ, 511, 118

Kobulnicky, H. A., Kennicutt, R. C. Jr., \& Pizagno, J. L. 1999, ApJ, 514, 544

Kocevski, D., Modjaz, M., Bloom, J. S., et al. 2007, ApJ, 663, 1180

Kouveliotou, C., Meegan, C. A., Fishman, G. J., et al. 1993, ApJ, 413, 101

Kunth, D., \& Joubert, M. 1985, A\&A, 142, 411

Kunth, D., \& Schild, H. 1986, A\&A, 169, 71

Lamareille, F., Mouhcine, M., Contini, T., Lewis, L., \& Maddox, S. 2004, MNRAS, 350, 396

Le Floc'h, E., Duc, P.-A., Mirabel, I. F., et al. 2003, A\&A, 400, 499

Levesque, E. M., \& Kewley, L. J. 2007, ApJ, 667, 121

Levesque, E. M., Berger, E., Kewley, L. J., et al. 2010, AJ, 139, 694

Liang, Y. C., Hammer, F., Deng, L. C., et al. 2006, PABei, 24, 335

Liang, Y. C., Hammer, F., Yin, S. Y., et al. 2007, A\&A, 473, 411

MacFadyen, A. I., \& Woosley, S. E. 1999, ApJ, 524, 262

Margutti, R., Chincarini, G., Covino, S., et al. 2007, A\&A, 474, 815

Massey, P. 2003, ARA\&A, 41, 15

Massey, P., \& Conti, P. 1980, ApJ, 242, 638

Massey, P., \& Conti, P. 1983, PASP, 95, 440

McGaugh, S. S. 1991, ApJ, 380, 140

Melbourne, J., \& Salzer, J. J. 2002, AJ, 123, 2302

Oke, J. B., \& Sandage, A. 1968, ApJ, 154, 21

Osterbrock, D. E. 1989, Astrophysics of Gaseous Nebulae and Active Galactic Nuclei (Mill Valley, California: University Science Books)

Pagel, B. E., Edmunds, M. G., Blackwell, D. E., Chun, M. S., \& Smith, G. 1979, MNRAS, 189, 95

Petrovic, J., Langer, N., Yoon, S.-C., et al. 2005, A\&A, 435, 247

Pian, E., Mazzali, P. A., Masetti, N., et al. 2006, Nature, 442, 1011

Prochaska, J. X., Bloom, J. S., Chen, H.-W., et al. 2004, ApJ, 611, 200

Richer, M. G., \& McCall, M. L. 1995, ApJ, 445, 642

Salpeter, E. E. 1955, ApJ, 121, 161

Savaglio, S., Glazebrook, K., \& Le Borgne, D. 2009, ApJ, 691, 182

Schaerer, D., \& Vacca, W. D. 1998, ApJ, 497, 618

Schlegel, D. J., Finkbeiner, D. P., \& Davis, M. 1998, ApJ, 500, 525

Seaton, M. J. 1979, MNRAS, 187, 73

Shaw, R. A., \& Dufour, R. J. 1994, Astronomical Data Analysis Software and Systems III, ed. D. R. Crabtree, R. J. Hanisch, \& J. Barnes, ASP Conf. Ser., 61,327

Skillman, E. D., Kennicutt, R. C., Jr., \& Hodge, P. W. 1989, ApJ, 347, 875

Smith, L. F., Shara, M. M., \& Moffat, A. F. J. 1990, ApJ, 348, 471

Smith, L. F., Shara, M. M., \& Moffat, A. F. J. 1996, MNRAS, 281, 163

Soderberg, A. M., Kulkarni, S. R., \& Berger, E. 2004, ApJ, 606, 994

Sokolov, V. V., Fatkhullin, T. A., Castro-Tirado, A. J., et al. 2001, A\&A, 372, 438

Sollerman, J., Jaunsen, A. O., Fynbo, J. P. U., et al. 2006, A\&A, 454, 503

Stasińska, G. 2005, A\&A, 434, 507

Thöne, C. C., Fynbo, J. P. U., Östlin, G., et al. 2008, ApJ, 676, 1151

Tremonti, C. A., Heckman, T. M., Kauffmann, G., et al. 2004, ApJ, 613, 898

Torres, A. V., Conti, P. S., \& Massey, P. 1986, ApJ, 300, 379

Vacca, W. D., \& Conti, P. S. 1992, ApJ, 401, 543

van der Hucht, K. A., Conti, P. S., Lundström, I., et al. 1981, Space Sci. Rev., 28, 227

van Paradijs, J., Groot, P. J., Galama, T., et al. 1997, Nature, 386, 686

Vreeswijk, P. M., Galama, T. J., Owens, A., et al. 1999, ApJ, 523, 171

Wainwright, C., Berger, E., \& Penprase, B. E. 2007, ApJ, 657, 367

Wiersema, K., Savaglio, S., Vreeswijk, P. M., et al. 2007, A\&A, 464, 529

Woosley, S. E., \& Heger, A. 2006, ApJ, 637, 914

Woosley, S. E., Langer, N., \& Weaver, T. A. 1993, ApJ, 411, 823

Yin, S. Y., Liang, Y. C., Hammer, F., et al. 2007, A\&A, 462, 535

Yoon, S.-C., \& Langer, N. 2005, A\&A, 443, 643 